\title{
Yolk sac tumor in intraabdominal undescended testis in a child with normal serum alpha-fetoprotein: a rare case report
}

\author{
Archika Gupta ${ }^{1 *} \mathbb{D}$, Shiv Narain Kureel ${ }^{1}$, Shalini Bhalla², Anand Pandey ${ }^{1}$, Gurmeet Singh ${ }^{1}$, Rahul Kumar Rai ${ }^{1}$ \\ and Survesh Kumar Gupta'
}

\begin{abstract}
Background: Intraabdominal testicular tumors are rare in prepubertal children, and most of the cases reported are intraabdominal testicular teratoma. The present study reports the first case of intraabdominal testicular yolk sac tumor (YST) with normal preoperative serum alpha-fetoprotein levels and diagnosis confirmed on histopathology and immunohistochemistry.

Case Presentation: A 2-year-old boy presented with bilateral nonpalpable undescended testes (UDT) and abdominal mass. Preoperative serum alpha-fetoprotein was normal. Contrast-enhanced computed tomography (CECT) scan of whole abdomen revealed a heterogeneous mildly enhancing space-occupying lesion in midline and left side of pelvis, left intraabdominal testis and nonvisualization of right testis. During surgery, the mass was found to involve right testis with one turn of torsion of its pedicle. Derotation of testis was performed, and right radical orchiectomy was performed. Left orchiopexy was also performed at the same time. Histopathology and immunohistochemistry confirmed diagnosis of intraabdominal testicular YST. There was no recurrence or distant metastasis at 12-month follow-up after surgery.
\end{abstract}

Conclusion: In a case of nonpalpable UDT and abdominal mass/pain, one should always consider possibility of intraabdominal testicular tumor and should investigate the case with serum tumor markers and ultrasound/CECT abdomen. Further, histology of tumor helps in guiding treatment of condition.

Keywords: Case report, Intraabdominal testicular tumor, Testicular tumors, Testicular tumors in prepubertal children, Tumors in undescended testis

\section{Background}

Occurrence of testicular tumors in undescended testes (UDT) is a rare, but known complication. The incidence of testicular tumors in UDT is 3-8 times greater than in general population with normally descended testes, being most frequently associated with intraabdominal testis [1]. Majority of these testicular tumors in UDT occur in postpubertal cases [2, 3]. However, involvement of UDT with

\footnotetext{
*Correspondence: drarchika2006@rediffmail.com

1 Department of Pediatric Surgery, King George's Medical University, Lucknow, UP 226003, India

Full list of author information is available at the end of the article
}

tumor in prepubertal children is very rare, particularly in intraabdominal testis [2, 3]. Majority of intraabdominal testicular tumors were teratomas with only four cases of yolk sac tumor (YST) in intraabdominal testis reported in the literature [2-5]. But, no case of intraabdominal/testicular YST with normal preoperative serum alpha-fetoprotein has been reported in the literature to the best of our knowledge.

We report the first case of intraabdominal testicular YST in a 2-years-old boy presenting with bilateral nonpalpable UDT, abdominal mass, and normal serum alpha-fetoprotein. 


\section{Case Presentation}

A 2-year-old boy was admitted with complaints of bilateral empty scrotal sacs since birth, lower abdominal lump for one month, and mild abdominal discomfort for 15 days. No family history of similar complaints was present. There was no history of recurrent abdominal pain, fever, vomiting, constipation, diarrhea, hematuria, dysuria, malena, or any limb weakness. On examination, a $6.8 \times 5.8 \mathrm{~cm}$ mildly tender, ovoid, firm lump with smooth surface and well-defined margins was palpable in suprapubic region extending up to umbilicus and left iliac region (Fig. 1a). No other lump or organomegaly was present. Bilateral scrotal sacs were empty, and both testes were nonpalpable. Serum tumor markers estimation revealed normal serum alpha-fetoprotein by chemiluminescence microparticular assay $(2.09 \mathrm{IU} / \mathrm{ml})$, mildly raised serum lactate dehydrogenase by kinetic chemistry L-lactate to pyruvate conversion by electron acceptor method (495.3 U/L), and normal beta human chorionic gonadotropin by chemiluminescence microparticular assay $(1.15 \mathrm{mIU} / \mathrm{ml})$. Urine was negative for vanillylmandelic acid and homovanillic acid by ELISA method. X-ray abdomen and chest was normal. Ultrasound whole abdomen with bilateral scrotum revealed nonvisualization of testes in both scrotum and inguinal regions, and a well-defined heterogeneous solid-cystic mass with internal echoes of size $6.6 \times 5.9 \mathrm{~cm}$ in pelvis above and left to bladder and anterior to iliac vessels but separate from these structures. Left testis was seen near left deep inguinal ring but right testis was not visualized. Contrast-enhanced computed tomography (CECT) scan of whole abdomen revealed bilateral UDT with left testis intraabdominally adjacent to left deep inguinal ring, nonvisualization of right testis, and a heterogeneous mildly enhancing space-occupying lesion measuring $6.7 \times 5.6 \times 4.8 \mathrm{~cm}$ in midline and left side of pelvis above the bladder but separate from it (Fig. 1b). Clinical diagnosis of bilateral nonpalpable undescended testes with intraabdominal lump probably due to testicular tumor was made. The patient underwent an exploratory laparotomy (EL) through pfannenstiel incision. EL revealed an ovoid intraabdominal mass, over which small bowel was adhered, in midline in pelvis (Fig. 2a). Adhesiolysis revealed right intraabdominal testicular mass with one turn torsion of right spermatic cord that was derotated (Fig. 2b). Left UDT was seen near left deep inguinal ring. Retroperitoneal lymph nodes were not enlarged. Radical orchiectomy was performed after ligation of right spermatic cord. Left orchiopexy was also performed at the same time. The right testicular mass specimen was sent for histopathologic examination (HPE). Grossly, right testicular mass specimen measured $6.8 \times 5.5 \times 4.8 \mathrm{~cm}$ with smooth and congested outer surface. Epididymis measuring $3 \times 2 \times 1.5 \mathrm{~cm}$, vas deferens and testicular

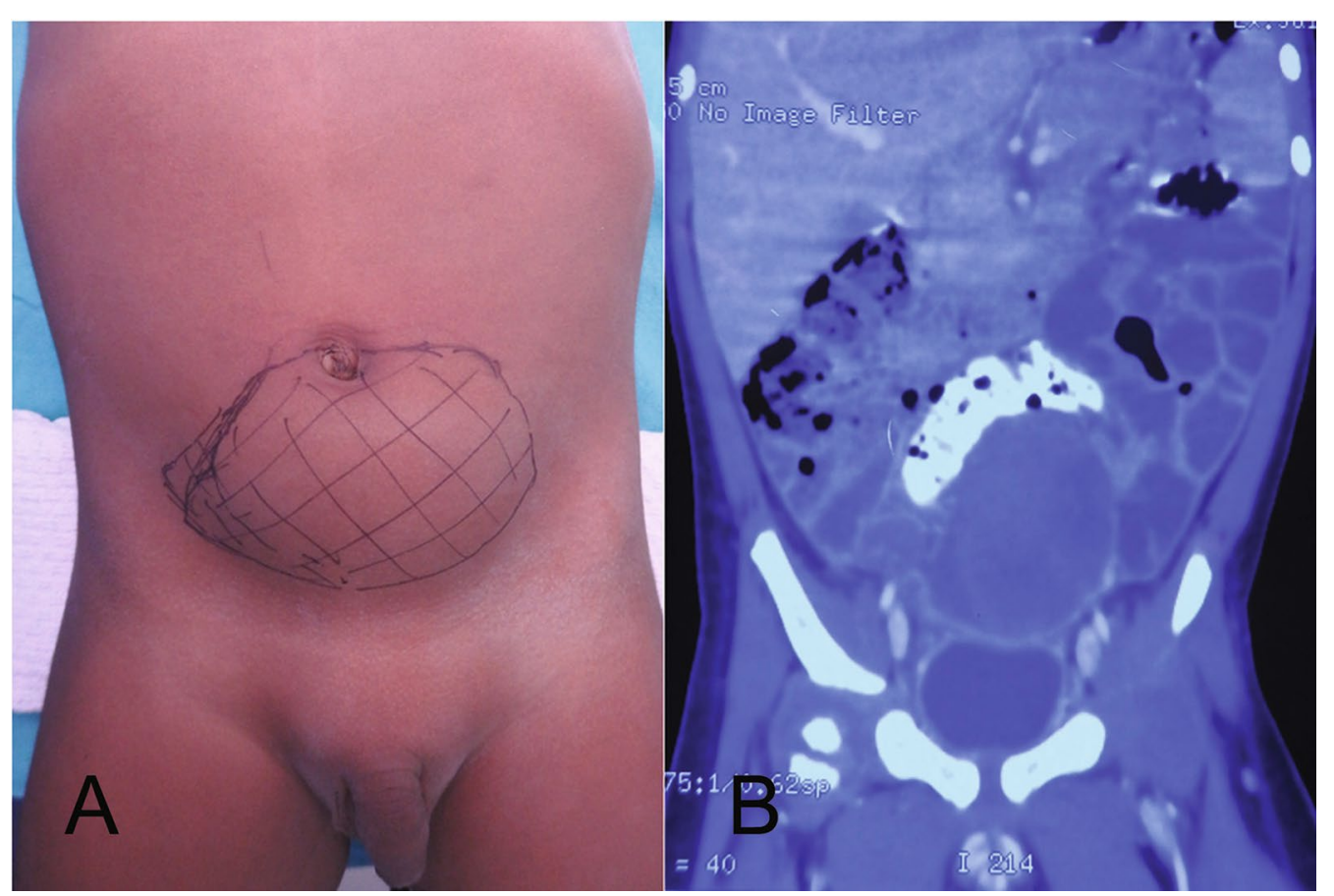

Fig. 1 a clinical picture of patient showing bilateral nonpalpable undescended testes in both scrotum and inguinal regions and a $6.8 \times 5.8 \mathrm{~cm}$ suprapubic lump, b CECT abdomen coronal section showing a heterogeneous mass in pelvis above the bladder and in midline 


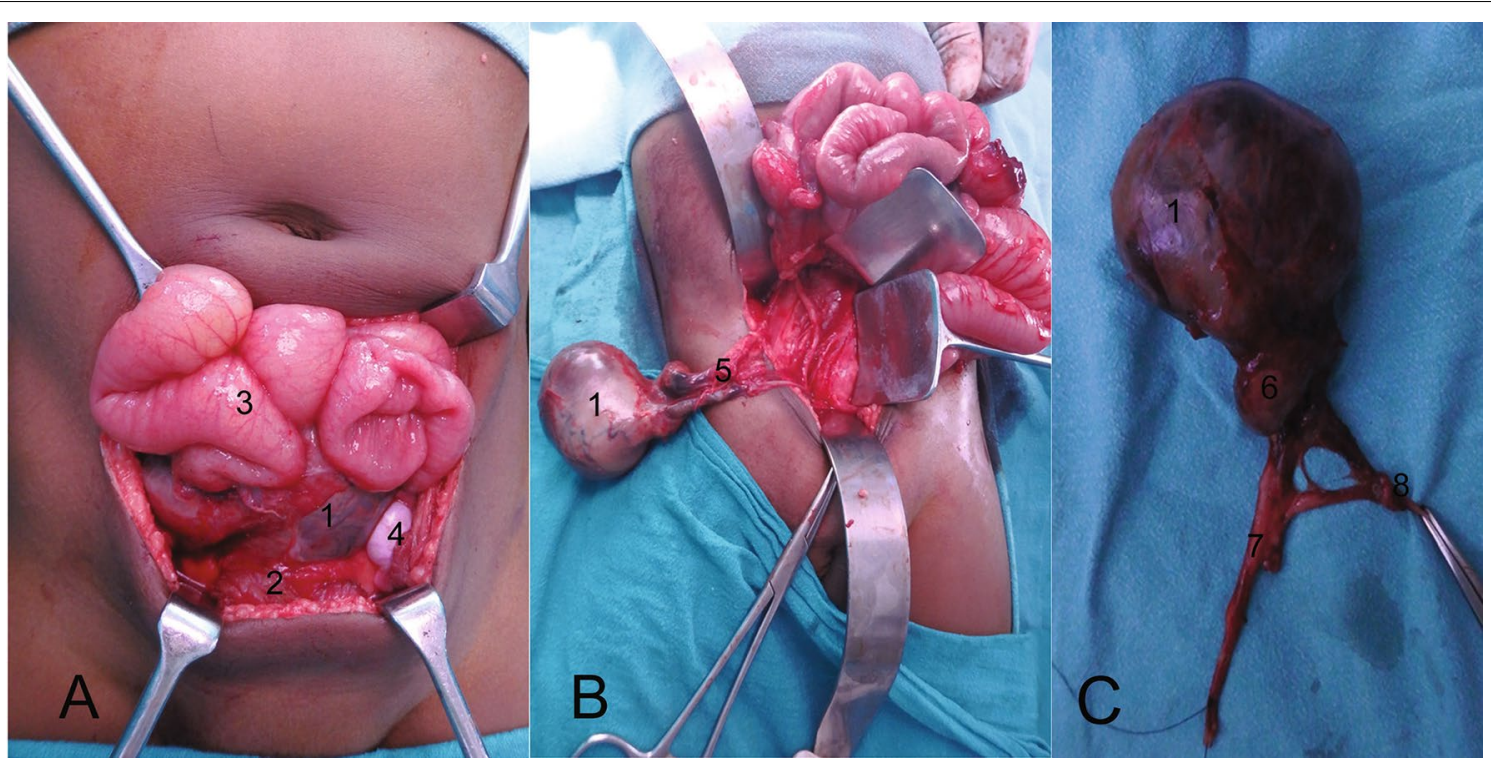

Fig. 2 a Operative picture showing intraabdominal mass (1) in pelvis above bladder (2). Over the mass (1), small bowel (3) is seen adhered. Left testis (4) is also seen intraabdominally in left side of pelvis proximal to deep inguinal ring, $\mathbf{b}$ operative picture showing right testicular mass (1) after adhesiolysis. Right testicular mass (1) had been derotated. Line of demarcation (5) is seen at spermatic cord at site of torsion after its derotation with congested testicular mass, testicular vessels, and vas deferens distal to site of torsion, c gross specimen of right testis with attached epididymis (6), vas deferens (7), and testicular vessels (8) after radical orchiectomy that have congested and smooth outer surface

vessels were attached to mass (Fig. 2c). Cut surface was firm showing grayish-brown foci of testicular tissue and replacement of near whole of testicular parenchyma by hemorrhagic and necrotic area (Fig. 3a). HPE revealed an encapsulated lesion with predominantly large area of hemorrhagic necrosis and interstitial hemorrhage interspersed with small areas of variably sized atypical cells with comprising of round to anisomorphic nuclei and moderate to abundant vacuolated cytoplasm, areas of mitosis at places, and multiple dilated congested vascular channels filled with blood. Areas of hemosiderin deposition were also seen (Fig. 3b, c). Sections from vas deferens and epididymis showed no evidence of tumor. Immunohistochemistry showed positivity for alpha-fetoprotein in tumor cells (Fig. 3d). After HPE and immunohistochemistry, final diagnosis of right-sided intraabdominal testicular YST with intratumoral necrosis was made. Serum tumor markers in postoperative period and at monthly follow-up remained within normal range and there was no recurrence or distant metastasis at 12-month followup after surgery.

\section{Discussion}

Testicular tumors in children are rare accounting for $1-2 \%$ of all pediatric solid tumors with an incidence of $0.5-2.0 / 100,000$ children and adolescents $[2,5,6]$. About $10 \%$ of testicular tumors are associated with UDT, and association between UDT and malignancy has been well established [7]. Earlier risk of developing testicular tumor in UDT had been considered to be 30-50 times greater than in the general population being highest with IAT intraabdominal testis [8], but recently, it has been estimated as 3-8 times higher than in the general population and most commonly seen in intraabdominal testis [1]. Testicular tumors in UDT usually occur at the same age as testicular tumors in normally descended testes, i.e., 20-40 years [2-4]. However, testicular tumors arising in UDT are rare in prepubertal children, particularly in intraabdominal testis $[2,3]$.

Since its first report in 1941 to 2015, 38 cases of intraabdominal testicular tumors in prepubertal children had been reported [2,3]. Most of the intraabdominal testicular tumors in prepubertal children were teratomas in contrast to intraabdominal testicular tumors in adolescents and adults in whom seminoma is the most common testicular tumors [2,3]. Malignant tumors in intraabdominal testis in prepubertal children are very unusual. We have found only four cases of intraabdominal testicular YST in prepubertal children. YSTs produce alpha-fetoprotein in very high concentrations and can be diagnosed by measuring raised serum alpha-fetoprotein levels [6]. However, our case is the first case of intraabdominal testicular YST because despite being YST, preoperative serum alpha-fetoprotein was normal. This could be explained by replacement of majority of testicular tissue by necrosis and hemorrhage as seen in HPE 

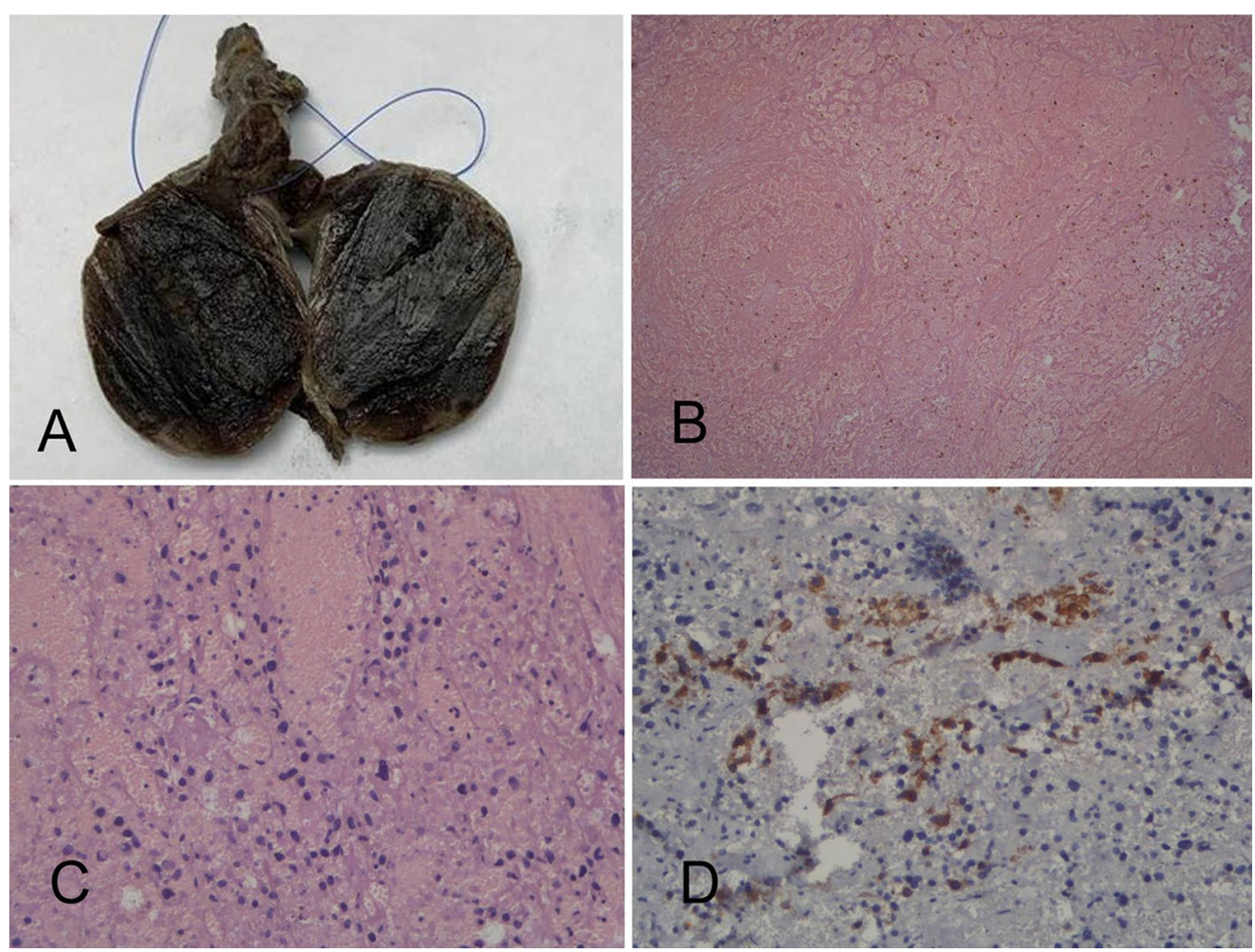

Fig. 3 a Cut section of testicular specimen displaying brownish-red diffuse hemorrhagic and necrotic areas replacing nearly whole of testicular parenchyma along with grayish-brown foci of testicular tissue, $\mathbf{b}$ and $\mathbf{c}$ low-power view of microscopic examination (H\&E-100 x) showing diffuse hemorrhagic necrosis of testicular parenchyma with focal interspersed viable tumor cells (d) Immunohistochemistry showing positivity for AFP in tumor cells $(\times 100)$

due to the presence of torsion in our case. Torsion might have resulted in death of most of the right intraabdominal testicular YST tissue due to occlusion of blood supply to right testis and resulted in normal preoperative serum alpha-fetoprotein.

Testicular tumors in intraabdominal testis in prepubertal children can present either as asymptomatic testicular mass detected during evaluation of nonpalpable UDT or with symptoms like abdominal pain or abdominal lump associated with mid or severe pain, nausea, vomiting or low-grade fever due to torsion, hemorrhage, or rupture of intraabdominal testicular tumors $[2,3,9,10]$. Imaging studies such as ultrasound or CECT abdomen can be useful adjuncts in making diagnosis of intraabdominal testicular tumors in both asymptomatic and symptomatic cases with nonpalpable UDT [2, 3, 9]. Role of laparoscopy has also been described in both diagnosis and treatment for intraabdominal testicular tumors [10]. Torsion had been reported to occur in about $60 \%$ of cases of intraabdominal testicular tumors in prepubertal children $[2,3,10]$; similarly, our case too had torsion at the time of laparotomy, resulting in necrosis and hemosiderin deposition in HPE. Therefore, torsion testis forms an important differential diagnosis of intraabdominal testicular tumors; and exact diagnosis can be made after histopathologic examination and immunohistochemistry.

Majority of intraabdominal testicular tumors in prepubertal children are clinically stage I tumors which can be managed with radical orchiectomy and surveillance with frequent measurement of tumor markers and thoracic and abdominal imaging similar to those in descended testes in prepubertal children $[2,5,6]$. Therefore, we have also performed only right radical orchiectomy and frequent monitoring with estimation of tumor markers and imaging studies and noticed favorable outcome with normal serum alpha-fetoprotein and no recurrence or distant metastasis of tumor at 12-month follow-up after discharge.

\section{Conclusion}

In a child with nonpalpable UDT presenting with abdominal lump/pain, possibility of intraabdominal testicular tumor should always be included in differential diagnosis. Tumor markers can help in making the diagnosis, but are not always raised. Early ultrasound or CECT scan helps in early detection and diagnosis of an intraabdominal 
testicular tumor that can aid in planning of optimum treatment for favorable outcome.

\section{Abbreviations}

UDT: Undescended testes; YST: Yolk sac tumor; CECT: Contrast enhanced computed tomography; EL: Exploratory laparotomy; HPE: Histopathologic exam.

\section{Acknowledgements}

Not applicable.

\section{Authors' contributions}

AG, SNK, SB, and GS were involved in substantial contributions to conception of the case report and acquisition of data. GS, RKR, and SKG were involved in substantial contributions to literature search. AG was involved in substantial contribution to writing of the paper manuscript with revision. SNK and AP were involved in substantial contributions to critical revision. GS, RKR, and SKG followed the patient postoperatively under supervision of SNK, AG, and AP. GS, RKR, and SKG followed the patient postoperatively under supervision of SNK, AG, and AP. All authors have read and approved the final version of the manuscript.

\section{Funding}

None.

\section{Availability of data and materials}

The data used in the current study are with the corresponding author and are available on reasonable request.

\section{Ethics approval and consent to participate}

Not applicable (Approval from an institutional board review is not required for a case report).

\section{Consent for publication}

Written informed consent had been taken from both parents for publication of this case report and corresponding images.

\section{Competing interests}

The authors declare that they have no competing interests.

\section{Author details}

${ }^{1}$ Department of Pediatric Surgery, King George's Medical University, Lucknow, UP 226003, India. ${ }^{2}$ Department of Pathology, King George's Medical University, Lucknow 226003, India.
Received: 30 July 2020 Accepted: 9 November 2020

Published online: 09 December 2020

\section{References}

1. Wood HM, Elder JS (2009) Cryptorchidism and testicular cancer: separating fact from fiction. J Urol 181:452-461

2. Tanaka N, Yoneda A, Fukuzawa M (2009) Mature teratoma arising from an intraabdominal testis in a 2-month-old boy: case report and review of intraabdominal testicular tumors in children. J Pediatr Surg 44:E15-E18

3. Mittal D, Agarwala S, Yadav DK, Pramanik DD, Sharma MC, Bagga D (2015) Testicular tumors in undescended testes in children below $5 \mathrm{y}$ of age. Indian J Pediatr 82:549-552

4. Cox L, Donald JC, Machin GA, Popkin JS, Zacks D (1988) Intraabdominal testis with yolk sac tumour in a 2-year-old child. J Pediatr Surg 23:775-776

5. Hirayama Y, Kubota M, Imamura M, Imai C, Okuyama N, Tsukada M et al (2009) A 2-year-old boy with a stage III yolk sac tumor occurring in an intra-abdominal retained testis. J Pediatr Surg 44:2395-2398

6. Ahmed HU, Arya M, Muneer A, Mushtaq I, Sebire NJ (2010) Testicular and paratesticular tumours in the prepubertal population. Lancet Oncol 11:476-483

7. Abratt RP, Reddi VB, Sarembok LA (1992) Testicular cancer and cryptorchidism. Br J Urol 70:656-659

8. Batata MA, Whitemore WF, Hilaris BS, Whitmore WF, Golbey RB (1982) Testicular cancer in cryptorchids. Cancer 49:1023-1030

9. Yama B, Georgioua NA, Khullar P, Corenc CV, Katz DS (2010) RadiologyPathology Conference: mature teratoma arising from an intra-abdominal undescended testis in a 7-month-old infant. Clin Imaging 34:466-471

10. Yada K, Ishibashi H, Mori H, Shimada M (2017) Laparoscopic resection of prenatally detected intra-abdominal testicular teratoma: Report of a neonatal case. J Pediatr Surg Case Rep 23:43-45

\section{Publisher's Note}

Springer Nature remains neutral with regard to jurisdictional claims in published maps and institutional affiliations.

\section{Submit your manuscript to a SpringerOpen ${ }^{\circ}$ journal and benefit from:}

- Convenient online submission

- Rigorous peer review

- Open access: articles freely available online

- High visibility within the field

- Retaining the copyright to your article

Submit your next manuscript at $\boldsymbol{\nabla}$ springeropen.com 